\title{
Rapid charge redistribution leading to core hollowing in a high-intensity ion beam
}

\author{
K. Ruisard ๑* and A. Aleksandrov@ \\ Oak Ridge National Laboratory, Oak Ridge, Tennessee 37830, USA
}

(Received 18 August 2020; accepted 16 December 2020; published 12 January 2021)

\begin{abstract}
Recently, the first direct measurement of a full 6D accelerator beam distribution was reported [B. Cathey, S. Cousineau, A. Aleksandrov, and A. Zhukov, Phys. Rev. Lett. 121, 064804 (2018)]. That work observed a correlation between energy and transverse coordinates, for which the energy distribution becomes hollowed near the transverse core. This hollowing is obscured when the 6D phase space is projected onto one- and two-dimensional axes. This article illustrates how a similar structure emerges from simulation of an initially uncorrelated, high density bunched beam as the result of velocity perturbation from initial nonlinear space charge forces. This phenomenon has not been widely recognized in accelerator systems, but parallels can be drawn to observations of laser-ionized nanoclusters and electron sources for diffraction. While this effect provides insight into the origin of the measured core correlation, it does not provide a complete description. A better reproduction of the measured structure can be obtained via selfconsistent simulation through the radio-frequency quadrupole.
\end{abstract}

DOI: 10.1103/PhysRevAccelBeams.24.014201

\section{INTRODUCTION}

Understanding beam dynamics in the early stages of capture and acceleration is crucial in high-intensity accelerators. Space charge dynamics in low- to medium-energy transport are suspected to initiate halo formation [1,2], causing uncontrolled losses at higher-power stages. Improved loss mitigation requires predictive capability of accelerator models. At present, no model has been shown to deliver accurate representation of the loss-prone beam tails and halo. This shortcoming has been attributed to incomplete information of the initial distribution [3]. Work at the Spallation Neutron Source (SNS) Beam Test Facility (BTF) addresses this gap by undertaking complete characterization of the $6 \mathrm{D}$ beam distribution downstream of the radiofrequency quadrupole (RFQ) [4].

The measurements in [4] revealed a hollowed, bimodal energy distribution for particles near the beam core. This feature, which was observed to scale with beam current, was characterized as new and unexpected. However, as this article will describe, this hollowing may be understood as a signature of charge redistribution. In this context, the conditions leading to longitudinal core hollowing are understood to be generally present in high-intensity accelerator front ends such as the BTF.

\footnotetext{
"ruisardkj@ornl.gov
}

Published by the American Physical Society under the terms of the Creative Commons Attribution 4.0 International license. Further distribution of this work must maintain attribution to the author(s) and the published article's title, journal citation, and DOI.
Work in [4] showed that the hollow energy profile forms in simple particle-in-cell (PIC) simulations of the bunch in the medium energy beam transport (MEBT) section. This article extends that work by taking a closer look at the bunch evolution. It will be shown that the energy splitting leads to "pile up" of excess density at the beam edge as a result of an outwardly-propagating density perturbation. This perturbation is launched by nonlinear space charge in the initial distribution which causes the core to expand more rapidly than the edge.

The key to observing core hollowing is examining slices rather than full projections of the $6 \mathrm{D}$ phase space. As observed in [4], for a $40 \mathrm{~mA} \mathrm{BTF}$ beam the hollowed core distribution is obscured in the full projection, which has smooth and monotonically decreasing profiles. This illustrates that standard characterization of the 2D phase spaces may neglect significant core features, particularly for high-charge bunches $\left(\sim 6 \times 10^{8}\right.$ ions/bunch in the BTF). A method of visualizing higher-dimensional features of the beam distribution is by extracting slices from the full distribution and characterizing the resulting partial phase spaces. The slice-emittance approach has not previously been adopted for characterization of ion beams but is already established in the FEL community, as variation of transverse phase space along the bunch can reduce brightness of the emitted coherent radiation $[5,6]$.

As the demand for high current at high reliability grows, the details of core structure are expected to be important for accurately modeling downstream beam dynamics and losses. The risk of remaining blind to core structure is over-simplification; e.g., adopting a 6D Gaussian on the basis that $1 \mathrm{D}$ and $2 \mathrm{D}$ projections appear Gaussian. It will be 
shown that the bunch produced by self-consistent tracking of particles through the RFQ is distinctly non-Gaussian, containing core hollowing in qualitative agreement with observations.

The paper is organized as follows. Section II summarizes the relevant observations at the SNS BTF. Section III describes a mechanism for space charge driven hollowing observed in various charged particle systems. Then, Sec. IV examines particle-in-cell simulation of an initial Gaussian distribution in a model of the BTF MEBT. A bunch with excessive charge $\left(100 \mathrm{~mA}\right.$ or $1.6 \times 10^{9}$ ions $)$ is used to visualize this mechanism. Section V compares this simple model to output from RFQ simulations. Finally, Sec. VI addresses the implications to simulation and measurement of high-intensity bunched beams.

\section{OBSERVATIONS AT THE SNS BEAM TEST FACILITY}

As mentioned, in prior work direct measurement of the 6D distribution was demonstrated [4]. The importance of studying slices rather than full projections was a key insight from this study. The core distribution was characterized via partial projections, which represent only a fraction of particles selected by slices along one or more dimensions. The dimensionality of the partial projections is defined as the number of slices; higher dimensional slices increase the ratio of core to edge particles and reveal the core structure. In the nomenclature adopted here, slice: $x, x^{\prime}$ indicates slices applied in the $x$ and $x^{\prime}$ coordinates. Unless otherwise noted, slice: $x, x^{\prime}$ bisects the beam core, containing particles near $x=0$ and $x^{\prime}=0$. The width of the slice is determined by the physical width of slit apertures used in the measurement, $0.2 \mathrm{~mm}$. A 1D transverse slice contains $\sim 10 \%$ of total bunch particles, while a 4D transverse slice contains $\sim 0.01 \%$.

The observed nonlinear correlation is between the energy distribution and all transverse coordinates $\left(x, x^{\prime}, y, y^{\prime}\right)$. Figure 1 illustrates the observations. For core particles, the energy distribution is hollow. However, the fully projected beam distribution (as well as the partial projection with one core slice shown in Fig. 1) appears convex and single-peaked. As shown in Fig. 2, for coordinates near the transverse edge the energy profile is also convex and singlepeaked. The hollowed structure was observed to depend on space charge and is most pronounced at $40 \mathrm{~mA}$, the highest measured current. Due to the large linear phase-energy correlation, the energy hollowing is synonymous with spatial hollowing in the phase coordinate.

\section{SELF FIELD DRIVEN HOLLOWING IN CHARGED PARTICLE BUNCHES}

This phenomenon has been observed across diverse fields. In the field of laser-plasma interactions, laser ionization of a gaseous nanocluster results in Coulumb

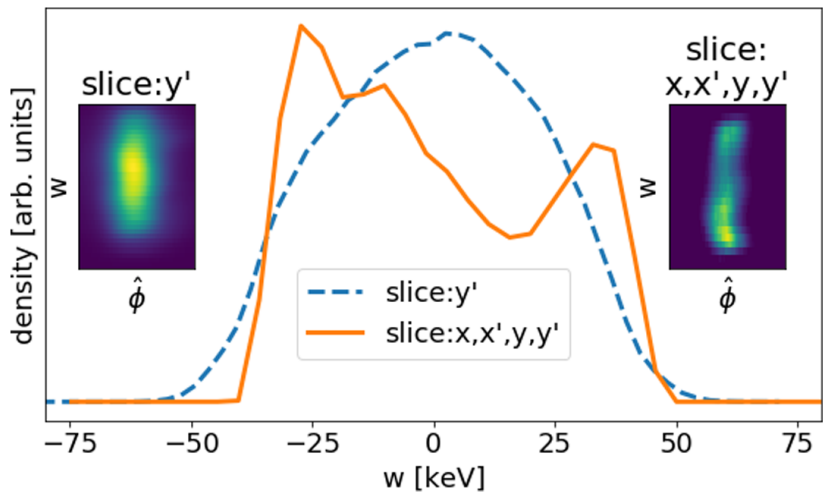

FIG. 1. Measured energy distribution for two levels of partial projection: a 1D core slice and 4D core slice. The longitudinal phase spaces are plotted in a shear-corrected plane, where $\hat{\phi}=\phi-\left\langle\frac{d \phi}{d w}\right\rangle w$. The beam current during the measurement is $21 \mathrm{~mA}$. This plot is consistent with results in [4], but reproduced with more recent measurements.

explosion (CE). A uniformly filled sphere is an equilibrium distribution in this expansion; however, even perturbative nonuniformity is shown to result in the formation of density rings on the outer edge of the expanding bunch as long as the density decreases from core to outer edge [7-9]. The initial nonuniformity results in core ions with higher outward velocity than edge ions. The system eventually reaches a crossover point, where inner particle trajectories catch up to outer particles and the radial velocity profile becomes multi-valued, leading to density pile-up and eventual shock formation.

This effect is also seen in the field of ultrafast electron diffraction (UED), where laser-driven photoemission creates a pancake-shaped bunch $(r \gg z)$ at the cathode surface. In this system, as bunch expands transversely a ringlike density spike appears at the edge [10,11]. Similar to the BTF observations, the hollow $x y$ profile is only visible for a slice through the longitudinal core, while

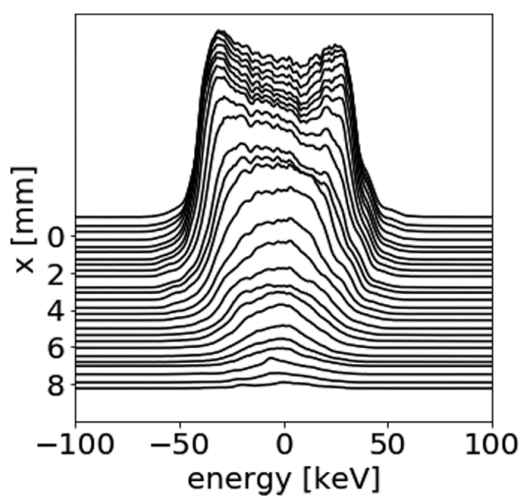

FIG. 2. Measured dependence of energy distribution of a 4D transverse slice: $x, x^{\prime}, y, y$ on transverse position. The slice is centered over the core in $x^{\prime}, y$ and $y^{\prime}$, but varied along $x$. The beam current during the measurement is $25 \mathrm{~mA}$. 
the fully-projected profile is uniform. Another similarity is the space charge dependence, as the ring only forms at electron densities relevant to next-generation UED sources.

In accelerators, charge redistribution is understood as the result of imbalance between the space charge force and the external focusing. In a linear focusing system, charge evolves toward a spatially uniform quasiequilibrium. Previous studies focus mainly on predicting the resulting emittance growth using Reiser's free energy model $[12,13]$. However, Wangler predicts emergence of a hollowed distribution prior to relaxation toward uniformity [14].

Wangler also notes that growth is enhanced by "transition toward stronger tune depression" [14]. This can be equivalently described as transition toward weaker focusing under which a compact bunch rapidly expands. Such is the case for injectors for bright electron beams. In modeling of rf photoinjectors [15], rapid transverse emittance growth was seen to correlate with longitudinal position and was accompanied by a density spike at the outer edge. Another parallel can be drawn to the application of laser-wakefield accelerators (LWFA) as electron injectors to colliders or FELs $[16,17]$, where very small emittance leads to significant space charge effect during bunch expansion between plasma boundary and rf structure. Modeling of this system reveals a similar deepening of a bimodal energy distribution with increased space charge [18].

As far as these authors are aware, the longitudinal hollowing of a bunched ion beam has not been previously studied. However, related effects in the transverse plane have been observed. In two University of Maryland experiments using low-energy electron beams to model ion beam dynamics, the transverse profiles developed visible density rings determined to be "the result of a strong beam-edge imbalance produced by an aperture in an expanding beam" $[19,20]$. Indistinguishable transverse hollowing may also be imposed by external rather than self-field nonlinearity; also in [19], some hollowed profiles are shown to originate from velocity perturbation due to field distortion at the cathode grid. Hollowed $x y$ profiles are observed in simulations of the International Fusion Materials Irradiation Facility (IFMIF) LEBT, which are traced back to nonlinear regions of the extraction electrodes [21]. Previous studies focused on uniformity at target have observed transverse hollowing from both self-fields and external nonlinearity $[22,23]$.

The cases discussed here share the characteristics of nonnegligible space charge forces, nonuniformity in initial charge distribution and a freely expanding bunch. In the following section it will be shown that this same phenomenon is driven in simulations of Gaussian bunches in the BTF MEBT, generating longitudinal distributions that resemble observations (Figs. 1 and 2).

\section{HOLLOWING OF AN INITIALLY GAUSSIAN MEBT BUNCH}

In the BTF MEBT, the beam at the RFQ exit is initially very compact. Within the first meter, the beam expands in all dimensions. Quadrupole focusing limits transverse size, but there is no longitudinal focusing. Therefore, it is consistent that hollowing is observed mainly along the longitudinal dimension.

Simulations of the MEBT are done with the PyORBIT code [24]. We assume a $2.5 \mathrm{MeV} H^{-}$bunch with $1.6 \times 10^{9}$ ions per bunch. This is equivalent to $100 \mathrm{~mA}$ average current, much higher than the nominal operating current of $40 \mathrm{~mA}$. This choice is made to decrease the time of onset and drive a more visible hollowing. The case at nominal SNS parameters is addressed in Sec. V.

First, a 1D longitudinal model is useful for illustrating the effect without applying high-dimensional slicing. An initial bunch of 200,000 macroparticles is seeded with a round and uniform transverse distribution of radius $1.3 \mathrm{~mm}$ (this is rms equivalent to the profile at the RFQ exit). The transverse profile is held constant in the simulation as if contained by constant transverse focusing. The initial longitudinal has zero emittance and the spatial distribution is Gaussian with $\sigma_{z}=16.5 \mathrm{~mm}$, about $10 \times$ longer than the expected length at the RFQ exit. In the 1D model, which takes an impedance-based approach to the space charge calculation, a perfectly conducting cylindrical pipe boundary is assumed at $2 \times$ the beam radius.

Figure 3 shows the longitudinal evolution in phase space, with the initial distribution shown in frame 0 . Energy $w$ is defined as $w=T-T_{0}$ for synchronous kinetic energy $T_{0}$. In frame 1, a nonlinear velocity kick from the space charge fields is visible and the profile has broadened. Here the
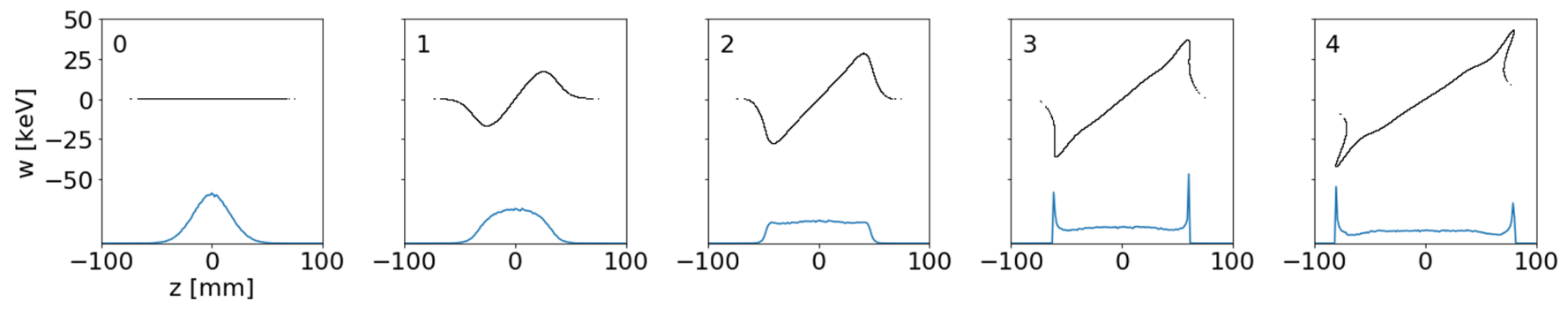

FIG. 3. Snapshots of phase space evolution in 1D longitudinal simulation at even intervals of 2.5 meter. A line-out of the spatial profile is also shown. 
TABLE I. Parameters for quadrupoles in first 1.3 meters of BTF MEBT.

\begin{tabular}{lcccc}
\hline \hline & $\mathrm{s}[\mathrm{m}]$ & $\int B \cdot d z[\mathrm{~T}]$ & $L_{\text {eff }}[\mathrm{cm}]$ & Polarity \\
\hline Quad 1 & 0.131 & 1.15 & 6.1 & + \\
Quad 2 & 0.314 & 1.36 & 6.6 & - \\
Quad 3 & 0.575 & 1.08 & 9.6 & + \\
Quad 4 & 0.771 & 0.61 & 9.6 & - \\
\hline \hline
\end{tabular}

TABLE II. rms parameters for $100 \mathrm{~mA}$ Gaussian initial distribution. Emittances are unnormalized.

\begin{tabular}{lrcc}
\hline \hline & $\mathrm{x}$ & $\mathrm{y}$ & $\mathrm{z}$ \\
\hline$\alpha$ & -2.0 & 2.0 & 0 \\
$\beta[\mathrm{mm} / \mathrm{mrad}]$ & 0.2 & 0.2 & $1.0(1.3 \mathrm{deg} / \mathrm{keV})$ \\
$\epsilon[\mathrm{mm}-\mathrm{mrad}]$ & 2.2 & 2.2 & $1.5(50 \mathrm{deg} \mathrm{keV})$ \\
\hline \hline
\end{tabular}

highest energy particles are found midway between the core and edge. By frame 2, the profile is more uniform. Frame 3 captures a crossover point, where inner particles

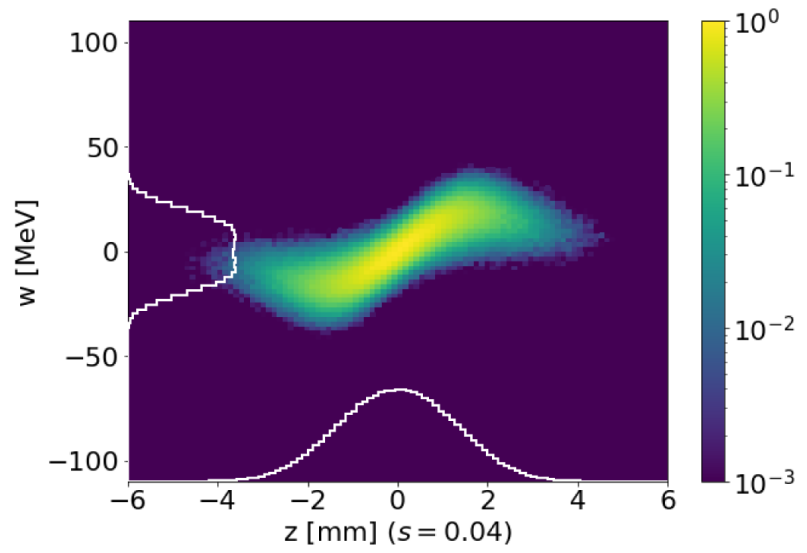

(a) Full projection at $s=4 \mathrm{~cm}$.

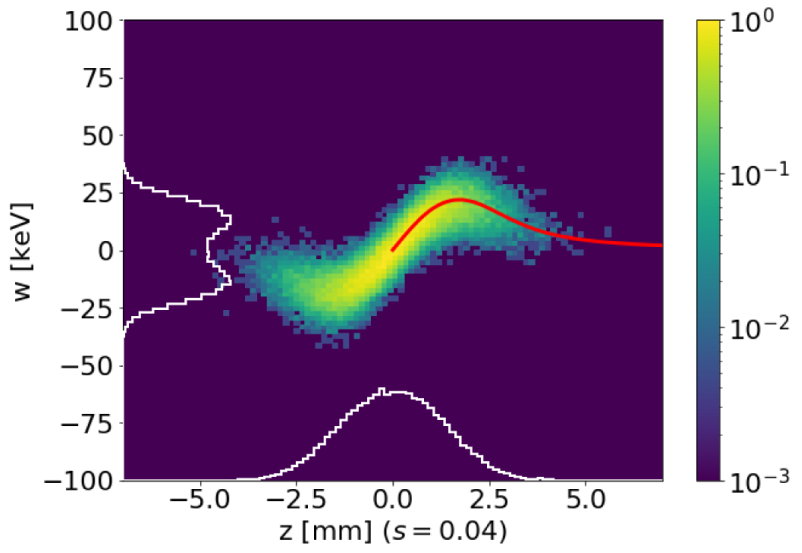

(c) Core-slice projection at $s=4 \mathrm{~cm}$. have caught up to outer particles. At this point the energy distribution $w(z)$ becomes multivalued and the expanding core folds over the tails. In the spatial profile, excess density has collected near the edges and two prominent peaks have formed. Between frames 3 and 4, the bunch expands with the two peaks "locked in" at the outer edge.

The same signatures can be seen in a 6D Gaussian beam when core slicing is applied. The same case is studied as in [4], which used the code PARMILA. The first meter includes four quadrupoles, which are modeled as hard-edged elements with the parameters reported in Table I. A Gaussian beam with Twiss parameters shown in Table II is seeded at the plane of the RFQ exit. These parameters were chosen to be near the rms parameters expected from the SNS RFQ with two notable differences. In addition to increasing the current to $100 \mathrm{~mA}$, the longitudinal emittance is half the design value of 100 deg-keV, which further accelerates the redistribution.

The simulation bunch is modeled with 500,000 macroparticles. This is above the requirement for saturation of

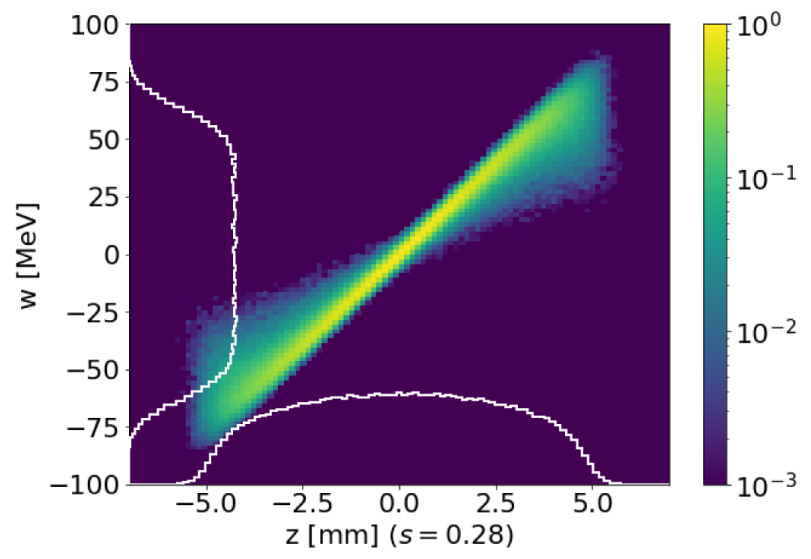

(b) Full projection at $s=28 \mathrm{~cm}$.

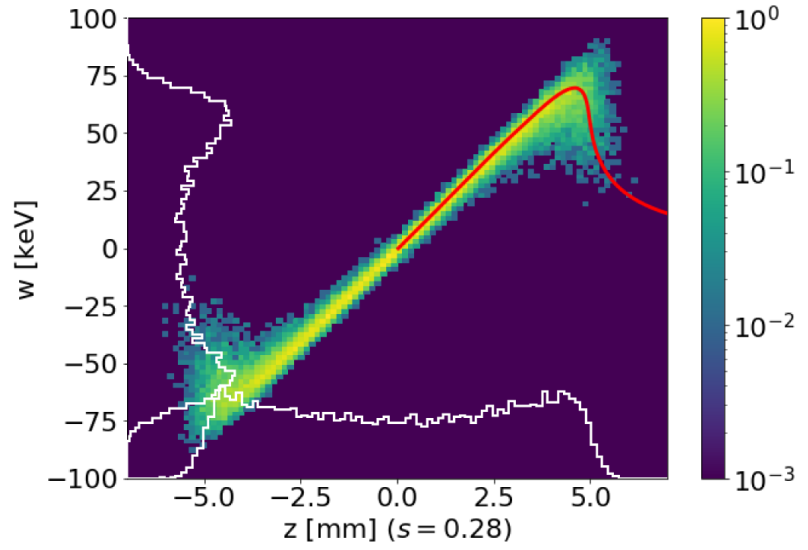

(d) Core-slice projection at $s=28 \mathrm{~cm}$.

FIG. 4. Evolution of the $100 \mathrm{~mA} 6 \mathrm{D}$ Gaussian beam in longitudinal phase space. Frames (a) and (b) show the full projection at $s=4 \mathrm{~cm}$ and $s=28 \mathrm{~cm}$. Frames (a) and (b) show a partial projection of the $10 \% 2 \mathrm{D}$ core slice in $x$ and $y$ at the same locations. The red line shows the evolution of a sequence of particles seeded along $z$, with initial $x, x^{\prime}, y, y^{\prime}, w=0$. 


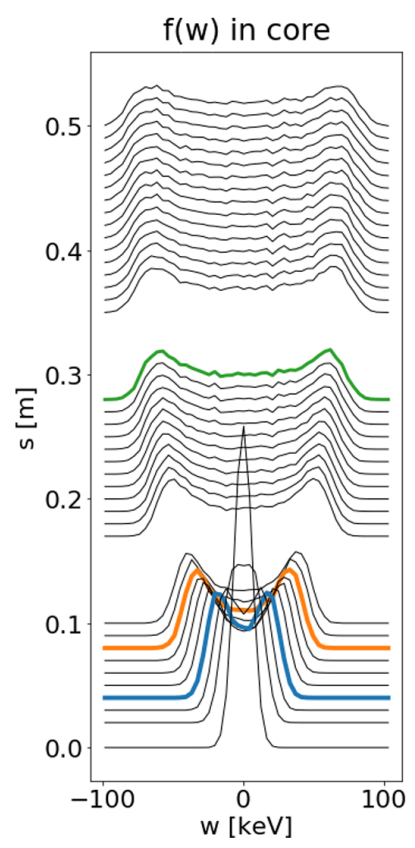

(a)

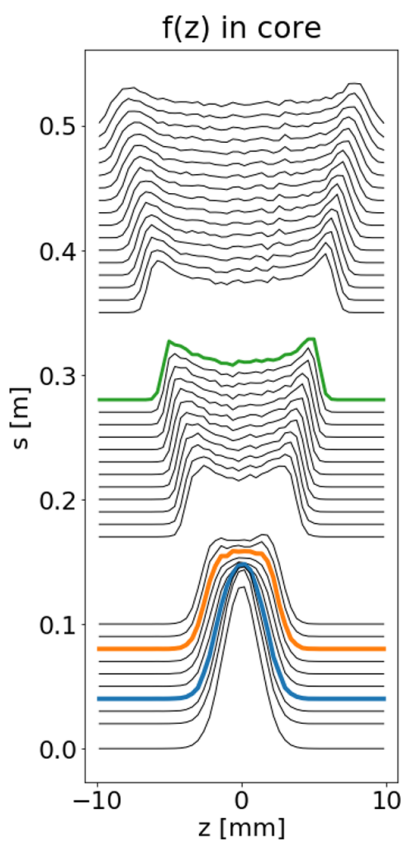

(b)
FIG. 5. Evolution of the (a) energy and (b) phase profiles in the $10 \%$ 2D core slice for the first 0.5 meters of evolution in the MEBT. The gaps indicate the quadrupole locations. Colored lines highlight the profile at $4 \mathrm{~cm}, 8 \mathrm{~cm}$ and $28 \mathrm{~cm}$.

$100 \%$ rms parameters, and is chosen to maintain good resolution of the phase space in core slices. The 3D PyORBIT space charge model uses a FFT Poisson solver with grid size $64 \times 64 \times 64$. The self fields are calculated every millimeter.

Snapshots of the longitudinal phase space during evolution are shown in Fig. 4(a) and 4(b). Consistent with the observations in [4], no hollowing is visible in the full projection. In Fig. 4(c), the initial velocity perturbation at $4 \mathrm{~cm}$ manifests as a broadening of the energy profile. Similarly, at $28 \mathrm{~cm}$ [Fig. 4(d)], the full projection has fairly uniform energy distribution and a convex spatial profile.

In order to visualize the emergence of core structure, bunch evolution is tracked for a partial distribution containing only particles near the transverse core in $x$ and $y$. As the transverse size varies during transport, the slice width is calculated dynamically. At each step, the slice width in each plane $[x, y]$ is set to contain $10 \%$ of particles in the 1D projections. As the beam broadens and the core redistributes, the number of particles in the slice is not fixed, and the 2D slice contains less than $10 \%$ of all particles. Snapshots of the phase space of the 2D core slices are shown in Fig. 4(c) and 4(d), which contain $7.8 \%$ and $4.4 \%$ of particles, respectively.

A more detailed view of the emergence of hollowing is shown in Fig. 5, which depicts the partial phase and energy profiles during the first $50 \mathrm{~cm}$ of transport. Within the first few steps, the effects of the space charge force are already

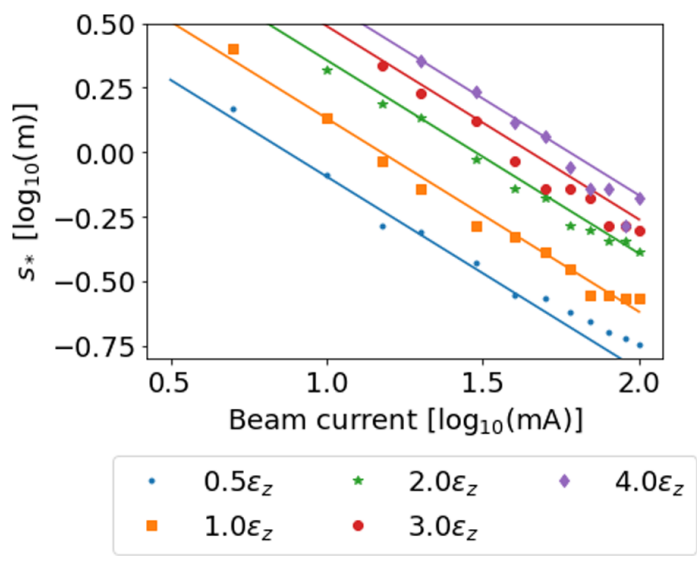

FIG. 6. Dependence of crossover point $s_{*}$ on average beam current for four different emittance values, where $\epsilon_{z}=50 \mathrm{deg} \mathrm{keV}$. The solid lines are defined by $s_{*}[m]=$ $10^{1.6}\left(\epsilon_{z}[\mathrm{~mm} \mathrm{mrad}] / I[\mathrm{~mA}]\right)^{0.75}$.

apparent as particles accelerate away from the core and the energy distribution broadens. At $4 \mathrm{~cm}$, splitting of the energy distribution $w(z)$ is visible [Fig. 4(c), blue curve in Fig. 5(b)]. Around $8 \mathrm{~cm}$, the energy perturbation has visibly changed the spatial profile [orange curve in Fig. 5(b)]. It appears broader, and shortly afterwards two distinct peaks appear, framing a hollowed core.

The crossover point occurs around $28 \mathrm{~cm}$, when the perturbed inner particles outrun edge particles. The foldover of the tails in phase space is very apparent in the core slice in Fig. 4(d). At the point, the edge has reached maximum steepness. After this point, indicated by the green curve in Figs. 5(a) and 5(b), the shape of the distribution locks in. The phase profile expands linearly, while the energy width saturates.

The location of the crossover point is a convenient metric to parametrize the speed of core redistribution. Qualitatively, the distribution evolves more rapidly and the resulting hollowing is deeper with increasing charge density. This occurs because the amplitude of the nonlinear velocity kick scales with charge. The crossover point is easily calculated by tracking a selection of particles initially distributed along $z$ and centered in the five other coordinates. The point at which the velocity profile $w(z)$ exceeds a threshold slope is tagged as the crossover. Figure 6 shows the crossover location for various currents and initial emittances. The fit lines follow the empirical relation, $s_{*}[m]=10^{1.6}\left(\epsilon_{z}[\mathrm{~mm} \mathrm{mrad}] / I[\mathrm{~mA}]\right)^{0.75} \quad($ emittance units are unnormalized).

Emittance growth also reflects the speed and magnitude of the core redistribution. Unlike the hollowing, this can readily be seen in the full distribution without taking core slices. For the $100 \mathrm{~mA}$ case discussed here (Table II), the $100 \% \mathrm{rms}$ emittance more than doubles in the first $20 \mathrm{~cm}$ of transport. Figure 7 compares emittance growth for different beam currents and fixed rms parameters (using the values in 


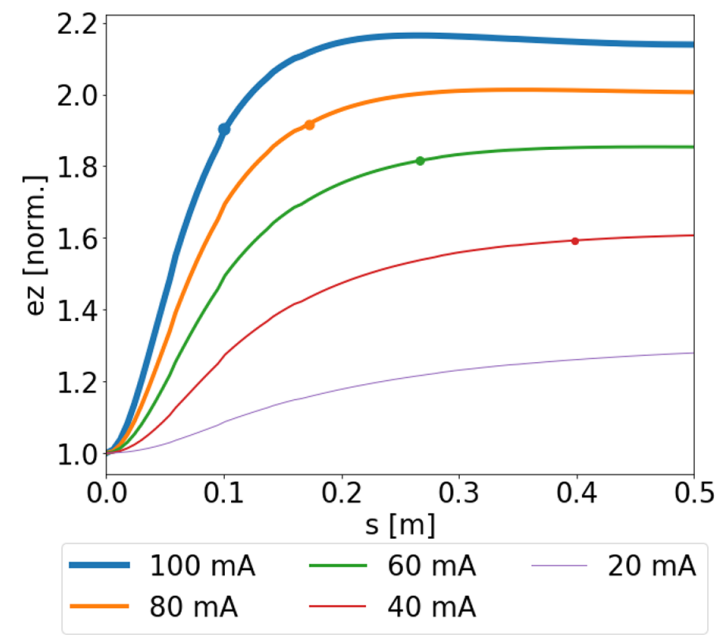

FIG. 7. rms emittance evolution for initially Gaussian beam with $\epsilon_{z}=50 \mathrm{deg} \mathrm{keV}$ and varying currents. The point on each curve indicates the crossover location.

Table II). As expected [12,14], emittance growth due to charge redistribution is rapid and very dependent on charge density. As charge density decreases, the magnitude of emittance growth decreases and the timescale increases. This mirrors a decrease in the depth of the hollowing as well as lengthening of the crossover time. Early rapid growth of the $100 \%$ emittance can be understood as a signature of the less-visible redistribution of the core density.

It should be noted that the behavior summarized in Figs. 6 and 7 is dependent on the transverse focusing (Table I). Transverse confinement enhances the longitudinal redistribution. We observed that in the absence of transverse focusing, when the bunch is allowed to freely expand in all directions, the hollowing is suppressed. The presence of a vertical waist before the first quadrupole also effects the redistribution, but this has not been studied in detail.
TABLE III. Output emittances from PARMTEQ simulation of RFQ. Emittances are unnormalized.

\begin{tabular}{lccc}
\hline \hline Output & $x$ & $y$ & $z$ \\
\hline$\alpha$ & -2.1 & 1.6 & 0.2 \\
$\beta[\mathrm{mm} / \mathrm{mrad}]$ & 0.19 & 0.14 & $0.6(0.8 \mathrm{deg} / \mathrm{keV})$ \\
$\epsilon[\mathrm{mm}-\mathrm{mrad}]$ & 3.34 & 3.35 & $3.95(132 \mathrm{deg} \mathrm{keV})$ \\
\hline \hline
\end{tabular}

\section{RELEVANCE TO MEASUREMENTS}

One notable discrepancy between the simulation and measurement is that a much higher average current $(100 \mathrm{~mA})$ is needed to drive visible hollowing than was available in measurements $(40 \mathrm{~mA})$. In addition, the Gaussian distribution expands symmetrically while the observed profile in Fig. 1 is consistently lopsided. These discrepancies can be explained by the fact that a Gaussian beam is a poor model for the $6 \mathrm{D}$ phase space at the RFQ output. A bunch generated by RFQ simulation already contains interplane correlations that also resemble the observed structure. This is explored in detail in [25], but repeated here for illustration.

The RFQ bunch is generated by PARMTEQ [26] simulation of the $402.5 \mathrm{MHz}$ SNS RFQ [27,28]. The $50 \mathrm{~mA}$ input beam has uniform phase, is single-valued in energy at $65 \mathrm{keV}$ and has a transverse Gaussian distribution. 5,000,000 macroparticles are used to keep good particle statistics in high-dimensional slices. In this case, the slice width is fixed and held equal to the slice width used in $6 \mathrm{D}$ phase space measurements. The output bunch has $41 \mathrm{~mA}$ average current with rms Twiss parameters as reported in Table III. Figure 8(a) shows the 4D partial core projection at the RFQ exit. A slightly hollowed core distribution is already visible.

Figure $8(\mathrm{~b})$ shows partial and full phase profiles after propagation in PyORBIT to the measurement plane (1.3 meters downstream of the RFQ). Figure 8(c) shows

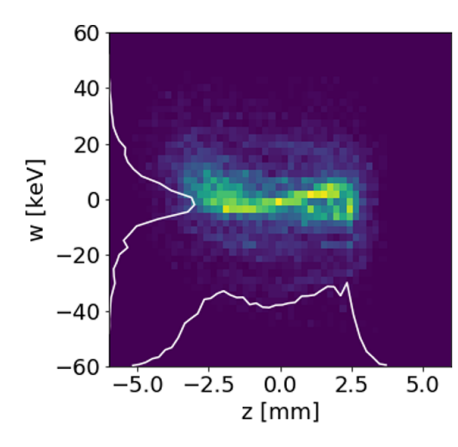

(a)

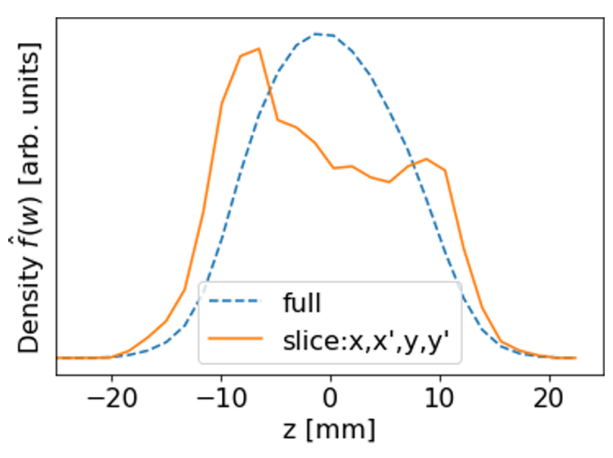

(b)

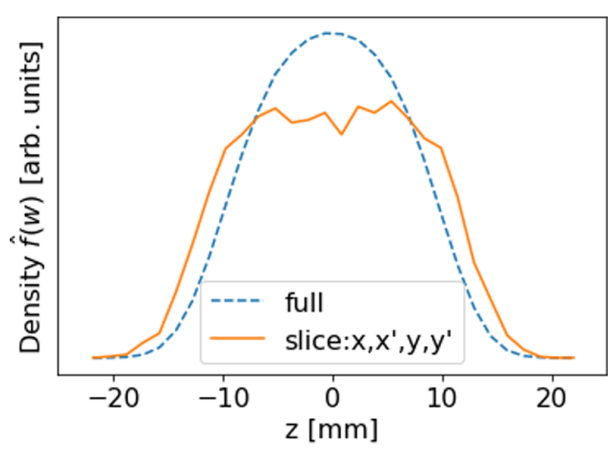

(c)

FIG. 8. (a) shows the 4D partial projection of the core of the $41 \mathrm{~mA}$ PARMTEQ simulated beam at RFQ output (slice contains $0.3 \%$ of total particles). (b-c) comparison of full and partial (4D core slice) MEBT phase profiles 1.3 meters downstream of the RFQ assuming two different initial conditions: (b) $41 \mathrm{~mA}$ PARMTEQ bunch and (c) $41 \mathrm{~mA}$ Gaussian. The partial phase profiles contain $0.2 \%$ and $0.3 \%$ of total particles, respectively. The initial MEBT distributions are rms equivalent. 
the same view assuming an rms equivalent Gaussian distribution at the RFQ exit. Although the fully projected profile is almost identical between the two cases, the partial profiles are distinct. The depth and width of the hollowing is very reduced for the $41 \mathrm{~mA}$ Gaussian when compared to the $100 \mathrm{~mA}$ case described previously. In comparison, the RFQ output bunch both has sharper features and qualitatively resembles the measured energy profile in Fig. 1.

Because of the core structure formed in the RFQ, the charge density of the core is already lower and more uniform than in the Gaussian bunch. As a result, no significant charge redistribution is observed in propagation through the MEBT. Simulated emittance growth of the RFQ bunch is only $2 \%$ within the first meter. As the PARMTEQgenerated bunch is understood to be more realistic than the Gaussian bunch, it is unlikely that the mechanism described here is excited in the experiment. However, observations confirm that the effect is space charge dependent. We predict the nonlinearities that lead to hollowing occur during the initial capture and bunch formation when space charge is most significant. Previous study of emittance growth in high-current RFQs noted transverse hollowing during the bunching stage [29], but did not observe a reciprocal effect in the longitudinal plane.

\section{DISCUSSION}

In summary, previous studies at the SNS BTF observed a nonlinear correlation between energy and transverse coordinates. This article describes a simple mechanism by which a Gaussian bunch can rapidly develop a similar structure during medium energy transport. The combination of nonlinear space charge fields and bunch expansion results in hollowing of the core density, an effect which has been seen in other high-density charged particle systems. While the hollowed core qualitatively resembles observations, self consistent simulation of the RFQ suggests that in the laboratory this structure develops before the MEBT.

Although insight from this mechanism will be useful in understanding the origin of core-hollowing within the RFQ, the dynamics may be quite different. The hollowing described here depends on bunch expansion: as the space charge density drops rapidly, the nonlinearity from the initial distribution is imprinted as a velocity perturbation. In contrast, within the RFQ the bunch is immersed in transverse and longitudinal focusing.

As this phenomenon is not expected to occur in a realistic MEBT bunch, effort should be made to avoid artificially inducing it in simulation. This study illustrates how the core distribution may be obscured in full projections and reinforces the need to create initial distributions with realistic, fully correlated $6 \mathrm{D}$ distributions. As demonstrated, spurious emittance growth can be driven when a convex initial distribution is inappropriately assumed. To avoid overestimating the space charge force, extra care must be taken to include the effects of upstream transverse-longitudinal coupling when generating high charge simulation bunches. The implications of this core structure for other space charge driven effects, such as halo formation and beam loss, is not yet understood and will be a focus in future studies.

\section{ACKNOWLEDGMENTS}

The authors are very grateful for assistance with PyORBIT and invaluable feedback on drafts provided by Jeff Holmes, Andrei Shishlo, and Sarah Cousineau. Thanks also to George Hine, who first recognized the similarity between the BTF observations and the physics of laser-ionized clusters. This research used resources at the Spallation Neutron Source, a DOE Office of Science User Facility operated by the Oak Ridge National Laboratory. This manuscript has been authored by UT-Battelle, LLC, under contract No. DE-AC05-00OR22725 with the U.S. Department of Energy (DOE). The U.S. government retains and the publisher, by accepting the article for publication, acknowledges that the US government retains a nonexclusive, paid-up, irrevocable, worldwide license to publish or reproduce the published form of this manuscript, or allow others to do so, for U.S. government purposes. DOE will provide public access to these results of federally sponsored research in accordance with the DOE Public Access Plan [30].

[1] R. L. Gluckstern, Analytic Model for Halo Formation in High Current Ion Linacs, Phys. Rev. Lett. 73, 1247 (1994).

[2] A. V. Fedotov, Mechanisms of halo formation, AIP Conf. Proc. 3, 3 (2003).

[3] J. Qiang, P. L. Colestock, D. Gilpatrick, H. V. Smith, T. P. Wangler, and M.E. Schulze, Macroparticle simulation studies of a proton beam halo experiment, Phys. Rev. Accel. Beams 5, 124201 (2002).

[4] B. Cathey, S. Cousineau, A. Aleksandrov, and A. Zhukov, First Six Dimensional Phase Space Measurement of an Accelerator Beam, Phys. Rev. Lett. 121, 064804 (2018).

[5] D. H. Dowell, P. R. Bolton, J. E. Clendenin, P. Emma, S. M. Gierman, W. S. Graves, C. G. Limborg, B. F. Murphy, and J. F. Schmerge, Slice emittance measurements at the SLAC gun test facility, Nucl. Instrum. Methods Phys. Res., Sect. A 507, 327 (2003).

[6] G. Dattoli, E. Sabia, C. Ronsivalle, M. Del Franco, and A. Petralia, Slice emittance, projected emittance and properties of the SASE FEL radiation, Nucl. Instrum. Methods Phys. Res., Sect. A 671, 51 (2012).

[7] A. E. Kaplan, B. Y. Dubetsky, and P. L. Shkolnikov, Shock Shells in Coulomb Explosions of Nanoclusters, Phys. Rev. Lett. 91, 143401 (2003).

[8] D. Murphy, R. W. Speirs, D. V. Sheludko, C. T. Putkunz, A. J. McCulloch, B. M. Sparkes, and R. E. Scholten, Detailed observation of space-charge dynamics using ultracold ion bunches, Nat. Commun. 5, 4489 (2014).

[9] M. Grech, R. Nuter, A. Mikaberidze, P. Di Cintio, L. Gremillet, E. Lefebvre, U. Saalmann, J. M. Rost, and S. 
Skupin, Coulomb explosion of uniformly charged spheroids, Phys. Rev. E 84, 056404 (2011).

[10] B. S. Zerbe, X. Xiang, C. Y. Ruan, S. M. Lund, and P. M. Duxbury, Dynamical bunching and density peaks in expanding Coulomb clouds, Phys. Rev. Accel. Beams 21, 064201 (2018).

[11] B. S. Zerbe and P. M. Duxbury, Density shocks in the relativistic expansion of highly charged one component plasmas, Phys. Rev. Accel. Beams 22, 114402 (2019).

[12] M. Reiser, Theory Des. Charg. Part. Beams, 2nd ed. (WileyVCH, Weinheim, 2008), Chap. 6, pp. 419-506.

[13] M. Reiser, C. R. Chang, D. Kehne, K. Low, T. Shea, H. Rudd, and I. Haber, Emittance Growth and Image Formation in a Nonuniform Space-Charge-Dominated Electron Beam, Phys. Rev. Lett. 61, 2933 (1988).

[14] T. P. Wangler, RF Linear Accel., 2nd ed. (Wiley-VCH, Weinheim, 2008), Chap. 9, pp. 282-340.

[15] S. G. Anderson and J. B. Rosenzweig, Nonequilibrium transverse motion and emittance growth in ultrarelativistic space-charge dominated beams, Phys. Rev. Accel. Beams 3, 094201 (2000).

[16] A. W. Chao, R. Pitthan, T. Tajima, and D. Yeremian, Space charge dynamics of bright electron beams, Phys. Rev. Accel. Beams 6, 024201 (2003).

[17] F. J. Grüner, C. B. Schroeder, A. R. Maier, S. Becker, and J. M. Mikhailova, Space-charge effects in ultrahigh current electron bunches generated by laser-plasma accelerators, Phys. Rev. Accel. Beams 12, 020701 (2009).

[18] G. Fubiani, J. Qiang, E. Esarey, W. P. Leemans, and G. Dugan, Space charge modeling of dense electron beams with large energy spreads, Phys. Rev. Accel. Beams 9, 064402 (2006).

[19] R. A. Kishek, S. Bernal, C. L. Bohn, D. Grote, I. Haber, H. Li, P. G. O'Shea, M. Reiser, and M. Walter, Simulations and experiments with space-charge-dominated beams, Phys. Plasmas 10, 2016 (2003).

[20] S. Bernal, R. Kishek, M. Reiser, and I. Haber, Observations and Simulations of Transverse Density Waves in a Collimated Space-Charge Dominated Electron Beam, Phys. Rev. Lett. 82, 4002 (1999).
[21] P. A. Nghiem, N. Chauvin, M. Comunian, O. Delferrière, R. Duperrier, A. Mosnier, C. Oliver, and D. Uriot, The IFMIF-EVEDA challenges in beam dynamics and their treatment, Nucl. Instrum. Methods Phys. Res., Sect. A 654, 63 (2011).

[22] D. Bruhwiler and Y.K. Batygin, Beam transport for uniform irradiation: nonlinear space charge and the effect of boundary conditions, Proc. IEEE Part. Accel. Conf. 5, 3254 (1995).

[23] Y. K. Batygin, Beam intensity redistribution in a nonlinear optics channel, Nucl. Instrum. Methods Phys. Res., Sect. B 79, 770 (1993).

[24] A. Shishlo, S. Cousineau, J. Holmes, and T. Gorlov, The particle accelerator simulation code PyORBIT, Procedia Comput. Sci. 51, 1272 (2015).

[25] K. Ruisard, A. Aleksandrov, S. Cousineau, A. Shishlo, V. Tzoganis, and A. Zhukov, High dimensional characterization of the longitudinal phase space formed in a radio frequency quadrupole, Phys. Rev. Accel. Beams 23, 124201 (2020).

[26] K. R. Crandall and T. P. Wangler, PARMTEQ_A beamdynamics code fo the RFQ linear accelerator, AIP Conf. Proc. 177, 22 (1988).

[27] S. Henderson, W. Abraham, A. Aleksandrov, C. Allen, J. Alonso, D. Anderson, D. Arenius, T. Arthur, S. Assadi, J. Ayers, P. Bach, V. Badea, R. Battle, J. Beebe-Wang, B. Bergmann et al., The Spallation Neutron Source accelerator system design, Nucl. Instrum. Methods Phys. Res., Sect. A 763, 610 (2014).

[28] A. Ratti, R. Digennaro, R. A. Gough, M. Hoff, R. Keller, K. Kennedy, R. Macgill, J. Staples, S. Virostek, and Yourd, The design of a high current, high duty factor RFQ for the SNS, in Proceedings of the European Particle Accelerator Conference, Vienna, 2000 (EPS, Geneva, 2000), pp. 495-497.

[29] T. P. Wangler, R. Mills, and K. R. Crandall, Emittance growth in intense beams, in Proceedings of the 1987 Particle Accelerator Conference (IEEE, Washington, DC, 1987), pp. 1007-1010.

[30] http://energy.gov/downloads/doe-public-access-plan. 